\title{
Absorption, Distribution and Toxicity Prediction of Curculigoside A and its Derivatives
}

\author{
Nursamsiar $^{1,2 *}$, Slamet Ibrahim ${ }^{1}$, Daryono H. Tjahjono ${ }^{1}$ \\ ${ }^{I}$ School of Pharmacy, Bandung Institute of Technology, Jalan Ganesha 10, Bandung 40132, Indonesia. \\ ${ }^{2}$ Sekolah Tinggi Ilmu Farmasi Makassar, Jl. P. Kemerdekaan Km 13,7 Daya, Makassar, Indonesia.
}

Received: 8 Oktober 2014 / Accepted: 30 November 2014

\begin{abstract}
:
Curculigoside A is a major bioactive phenolic glycoside of the medicinal plant of Curculigo orchioides. The present study was intended to obtain detail information of the pharmacokinetic properties including oral absorption, distribution, metabolism and toxicity of curculigoside A and its derivatives using in silico methods. Pharmacokinetic properties, absorption as well as distribution prediction using parameters of HIA (Human Intestinal Absorption), plasma protein binding, and permeability to Caco-2 cells were studied using the PreADMET and Toxtree package. The result showed that curculigoside A has absorption properties and permeability (permeability: middle) at medium level, and weakly bound to plasma proteins. Aglycone of curculigoside A was predicted to have good absorption properties and its permeability (permeability: middle) at medium level, and weakly bound to plasma proteins. Its derivatives, compounds of (2), (3), (4), (5), and (12), showed better absorption and distribution properties than that of curculigoside A. Toxicity prediction of curculigoside A and its derivatives showed no mutagenic or carcinogenic properties.
\end{abstract}

Key words: ADMET, curculigoside A, in silico, pharmacokinetic properties

\section{Introduction}

Curculigoside $\mathrm{A}$ is a phenolic glycoside compound isolated from the rhizome Curculigo orchioide [1]. It is the major bioactive compound present in Curculigo orchioides. It stimulates the secretion of estradiol by cultured primary granulosa cells and exhibits potent inhibitory activity against matrix metalloproteinase- 1 in cultured human skin fibroblasts $[2,3]$. It also attenuates human umbilical vein endothelial cell injury induced by $\mathrm{H}_{2} \mathrm{O}_{2}$ [4], up-regulates VEGF in MC3T3-E1 cells [5], and decreases infarct volume, alleviates cerebral damage, and reduces the expression of HMGB1 and phosphorylation of IkB- $\alpha$ and NF-kB in ischemic brain tissue [6].

In silico approaches are being used today in drug discovery to assess the ADME (Absorption, Distribution, Metabolism, Excretion) and toxicity properties of compounds at the early stages of discovery/development. The need for early consideration of ADME properties is also increasingly urgent because of the implementation of combinatorial chemistry and high- throughput screening, since this can generate vast numbers of potential lead compounds.

In the present research, Absorption, Distribution, Metabolism, Excretion dan Toxicity (ADMET) properties of curculigoside A and its aglicone, as well as it derivatives were predicted using Pre-ADMET software, and the toxicity prediction was generated using Toxtree software.

\section{Experimental}

The Pre-ADMET program was accessed at http://preadmet.bmdrc.org/. Curculigoside $\mathrm{A}$ and its derivatives were used in this study. The structure of all compounds were converted into molfile (*.mol). The program automatically calculated the predictive absorption for Caco-2 cell, and HIA (Human Intestinal Absorption), and distribution for plasma protein binding [7]. Prediction of toxicity properties was performed using Toxtree by applying and using Benigni/Bossa rulebase methods (for mutagenicity and carcinogenicity) [8].

\section{Results and Discussion}

The pharmacokinetic properties including absorption, distribution, metabolism and excretion of drug has an important role on its efficacy. Prediction of HIA is a major goal in the design, optimization, and selection of candidates for development as oral drugs, where the absorption of drug compounds in intestinal depends both on complex biological processes (including passive membrane penetration, active transport mechanisms and metabolism in the gastrointestinal tract).

\footnotetext{
*Corresponding author: Nursamsiar,

E-mail: n.siar@yahoo.co.id
} 
Tabel 1. Curculigoside A and its derivatives<smiles>[R]c1c([R])c([2H])c([R6])c(COC(=O)c2c([R6])c([R6])c([2H])c([2H])c2[R6])c1[R5]</smiles>

\begin{tabular}{|c|c|c|c|c|c|c|c|c|c|c|}
\hline \multirow{2}{*}{ No. } & \multirow{2}{*}{ Compound } & \multicolumn{9}{|c|}{ Substituens } \\
\hline & & $\mathrm{R}_{1}$ & $\mathrm{R}_{2}$ & $\mathrm{R}_{3}$ & $\mathrm{R}_{4}$ & $\mathrm{R}_{6}$ & $\mathrm{R}_{7}$ & $\mathrm{R}_{8}$ & $\mathrm{R}_{9}$ & $\mathrm{R}_{10}$ \\
\hline 1 & Curculigoside A & OGLY & $\mathrm{H}$ & $\mathrm{H}$ & $\mathrm{OH}$ & $\mathrm{OCH}_{3}$ & $\mathrm{H}$ & $\mathrm{H}$ & $\mathrm{H}$ & $\mathrm{OCH}_{3}$ \\
\hline 2 & Aglycone & $\mathrm{OH}$ & $\mathrm{H}$ & $\mathrm{H}$ & $\mathrm{OH}$ & $\mathrm{OCH}_{3}$ & $\mathrm{H}$ & $\mathrm{H}$ & $\mathrm{H}$ & $\mathrm{OCH}_{3}$ \\
\hline 3 & 2 & $\mathrm{H}$ & $\mathrm{OH}$ & $\mathrm{H}$ & $\mathrm{OH}$ & $\mathrm{OCH}_{3}$ & $\mathrm{H}$ & $\mathrm{H}$ & $\mathrm{H}$ & $\mathrm{OCH}_{3}$ \\
\hline 4 & 3 & $\mathrm{H}$ & $\mathrm{OH}$ & $\mathrm{H}$ & $\mathrm{OH}$ & $\mathrm{H}$ & $\mathrm{OH}$ & $\mathrm{H}$ & $\mathrm{OH}$ & $\mathrm{H}$ \\
\hline 5 & 4 & $\mathrm{OH}$ & $\mathrm{H}$ & $\mathrm{H}$ & $\mathrm{OH}$ & $\mathrm{H}$ & $\mathrm{OH}$ & $\mathrm{H}$ & $\mathrm{OH}$ & $\mathrm{H}$ \\
\hline 6 & 5 & $\mathrm{OH}$ & $\mathrm{H}$ & $\mathrm{H}$ & $\mathrm{OH}$ & $\mathrm{OCH}_{3}$ & $\mathrm{H}$ & $\mathrm{Cl}$ & $\mathrm{H}$ & $\mathrm{OCH}_{3}$ \\
\hline 7 & 6 & $\mathrm{OH}$ & $\mathrm{H}$ & $\mathrm{Cl}$ & $\mathrm{OH}$ & $\mathrm{OCH}_{3}$ & $\mathrm{H}$ & $\mathrm{Cl}$ & $\mathrm{H}$ & $\mathrm{OCH}_{3}$ \\
\hline 8 & 7 & $\mathrm{OH}$ & $\mathrm{H}$ & $\mathrm{Cl}$ & $\mathrm{OH}$ & $\mathrm{OCH}_{3}$ & $\mathrm{H}$ & $\mathrm{H}$ & $\mathrm{H}$ & $\mathrm{OCH}_{3}$ \\
\hline 9 & 8 & $\mathrm{OH}$ & $\mathrm{Cl}$ & $\mathrm{Cl}$ & $\mathrm{OH}$ & $\mathrm{OCH}_{3}$ & $\mathrm{H}$ & $\mathrm{Cl}$ & $\mathrm{H}$ & $\mathrm{OCH}_{3}$ \\
\hline 10 & 9 & $\mathrm{OH}$ & $\mathrm{H}$ & $\mathrm{Cl}$ & $\mathrm{OH}$ & $\mathrm{OCH}_{3}$ & $\mathrm{Cl}$ & $\mathrm{Cl}$ & $\mathrm{H}$ & $\mathrm{OCH}_{3}$ \\
\hline 11 & 10 & $\mathrm{OH}$ & $\mathrm{H}$ & $\mathrm{Cl}$ & $\mathrm{OH}$ & $\mathrm{OCH}_{3}$ & $\mathrm{H}$ & $\mathrm{Cl}$ & $\mathrm{Cl}$ & $\mathrm{OCH}_{3}$ \\
\hline 12 & 11 & $\mathrm{OH}$ & $\mathrm{Cl}$ & $\mathrm{Cl}$ & $\mathrm{OH}$ & $\mathrm{OCH}_{3}$ & $\mathrm{H}$ & $\mathrm{H}$ & $\mathrm{H}$ & $\mathrm{OCH}_{3}$ \\
\hline 13 & 12 & $\mathrm{OH}$ & $\mathrm{H}$ & $\mathrm{H}$ & $\mathrm{OH}$ & $\mathrm{OCH}_{3}$ & $\mathrm{Cl}$ & $\mathrm{Cl}$ & $\mathrm{H}$ & $\mathrm{OCH}_{3}$ \\
\hline 14 & 13 & $\mathrm{OH}$ & $\mathrm{H}$ & $\mathrm{H}$ & $\mathrm{OH}$ & $\mathrm{OCH}_{3}$ & $\mathrm{H}$ & $\mathrm{Cl}$ & $\mathrm{Cl}$ & $\mathrm{OCH}_{3}$ \\
\hline 15 & 14 & $\mathrm{OH}$ & $\mathrm{Cl}$ & $\mathrm{H}$ & $\mathrm{OH}$ & $\mathrm{OCH}_{3}$ & $\mathrm{H}$ & $\mathrm{Cl}$ & $\mathrm{H}$ & $\mathrm{OCH}_{3}$ \\
\hline 16 & 15 & $\mathrm{OH}$ & $\mathrm{CF}_{3}$ & $\mathrm{Cl}$ & $\mathrm{OH}$ & $\mathrm{OCH}_{3}$ & $\mathrm{H}$ & $\mathrm{H}$ & $\mathrm{H}$ & $\mathrm{OCH}_{3}$ \\
\hline 17 & 16 & $\mathrm{OH}$ & $\mathrm{H}$ & $\mathrm{H}$ & $\mathrm{OH}$ & $\mathrm{OCH}_{3}$ & $\mathrm{CF}_{3}$ & $\mathrm{Cl}$ & $\mathrm{H}$ & $\mathrm{OCH}_{3}$ \\
\hline 18 & 17 & $\mathrm{OH}$ & $\mathrm{CF}_{3}$ & $\mathrm{H}$ & $\mathrm{OH}$ & $\mathrm{OCH}_{3}$ & $\mathrm{H}$ & $\mathrm{Cl}$ & $\mathrm{H}$ & $\mathrm{OCH}_{3}$ \\
\hline 19 & 18 & $\mathrm{OH}$ & $\mathrm{H}$ & $\mathrm{Cl}$ & $\mathrm{OH}$ & $\mathrm{OCH}_{3}$ & $\mathrm{CF}_{3}$ & $\mathrm{H}$ & $\mathrm{H}$ & $\mathrm{OCH}_{3}$ \\
\hline 20 & 19 & $\mathrm{OH}$ & $\mathrm{CF}_{3}$ & $\mathrm{Cl}$ & $\mathrm{OH}$ & $\mathrm{OCH}_{3}$ & $\mathrm{H}$ & $\mathrm{Cl}$ & $\mathrm{H}$ & $\mathrm{OCH}_{3}$ \\
\hline 21 & 20 & $\mathrm{OH}$ & $\mathrm{H}$ & $\mathrm{Cl}$ & $\mathrm{OH}$ & $\mathrm{OCH}_{3}$ & $\mathrm{CF}_{3}$ & $\mathrm{Cl}$ & $\mathrm{H}$ & $\mathrm{OCH}_{3}$ \\
\hline 22 & 21 & $\mathrm{OH}$ & $\mathrm{CF}_{3}$ & $\mathrm{NO}_{2}$ & $\mathrm{OH}$ & $\mathrm{OCH}_{3}$ & $\mathrm{H}$ & $\mathrm{H}$ & $\mathrm{H}$ & $\mathrm{OCH}_{3}$ \\
\hline 23 & 22 & $\mathrm{OH}$ & $\mathrm{H}$ & $\mathrm{H}$ & $\mathrm{OH}$ & $\mathrm{OCH}_{3}$ & $\mathrm{CF}_{3}$ & $\mathrm{NO}_{2}$ & $\mathrm{H}$ & $\mathrm{OCH}_{3}$ \\
\hline 24 & 23 & $\mathrm{OH}$ & $\mathrm{H}$ & $\mathrm{Cl}$ & $\mathrm{OH}$ & $\mathrm{OCH}_{3}$ & $\mathrm{CF}_{3}$ & $\mathrm{NO}_{2}$ & $\mathrm{H}$ & $\mathrm{OCH}_{3}$ \\
\hline 25 & 24 & $\mathrm{OH}$ & $\mathrm{CF}_{3}$ & $\mathrm{NO}_{2}$ & $\mathrm{OH}$ & $\mathrm{OCH}_{3}$ & $\mathrm{H}$ & $\mathrm{Cl}$ & $\mathrm{H}$ & $\mathrm{OCH}_{3}$ \\
\hline 26 & 25 & $\mathrm{OH}$ & $\mathrm{H}$ & $\mathrm{NO}_{2}$ & $\mathrm{OH}$ & $\mathrm{OCH}_{3}$ & $\mathrm{CF}_{3}$ & $\mathrm{NO}_{2}$ & $\mathrm{H}$ & $\mathrm{OCH}_{3}$ \\
\hline 27 & 26 & $\mathrm{OH}$ & $\mathrm{H}$ & $\mathrm{NO}_{2}$ & $\mathrm{OH}$ & $\mathrm{H}$ & $\mathrm{H}$ & $\mathrm{NO}_{2}$ & $\mathrm{H}$ & $\mathrm{H}$ \\
\hline 28 & 27 & $\mathrm{H}$ & $\mathrm{OH}$ & $\mathrm{NO}_{2}$ & $\mathrm{OH}$ & $\mathrm{H}$ & $\mathrm{OH}$ & $\mathrm{NO}_{2}$ & $\mathrm{OH}$ & $\mathrm{H}$ \\
\hline 29 & 28 & $\mathrm{OH}$ & $\mathrm{H}$ & $\mathrm{H}$ & $\mathrm{OH}$ & $\mathrm{H}$ & $\mathrm{H}$ & $\mathrm{NO}_{2}$ & $\mathrm{H}$ & $\mathrm{H}$ \\
\hline 30 & 29 & $\mathrm{OH}$ & $\mathrm{H}$ & $\mathrm{H}$ & $\mathrm{OH}$ & $\mathrm{H}$ & $\mathrm{OH}$ & $\mathrm{NO}_{2}$ & $\mathrm{OH}$ & $\mathrm{H}$ \\
\hline
\end{tabular}


Table 2. Predictive absorption, distribution, and toxicity of curculigoside A and its derivatives.

\begin{tabular}{|c|c|c|c|c|c|c|}
\hline \multirow[b]{2}{*}{ No. } & \multirow[b]{2}{*}{$\begin{array}{l}\text { Name of } \\
\text { Compound }\end{array}$} & \multicolumn{2}{|r|}{ Absorption } & \multirow{2}{*}{$\begin{array}{c}\text { Distribution } \\
\begin{array}{c}\text { In vitro plasma } \\
\text { protein binding (\%) }\end{array}\end{array}$} & \multicolumn{2}{|c|}{ Toxicity risk parameters } \\
\hline & & $\begin{array}{l}\text { HIA } \\
(\%)\end{array}$ & $\begin{array}{l}\text { In Vitro Caco-2 } \\
\text { cell permeability } \\
\left(\mathrm{nm} \mathrm{sec}^{-1}\right)\end{array}$ & & Mutagenicity & carcinogenicity \\
\hline 1 & Tamoksifen & 100.00 & 49.54 & 94.74 & No risk & No risk \\
\hline 2 & Curculigoside A & 52.20 & 11.83 & 59.98 & No risk & No risk \\
\hline 3 & Aglycone & 92.53 & 20.78 & 85.75 & No risk & No risk \\
\hline 4 & Compound 2 & 92.53 & 15.56 & 86.98 & No risk & No risk \\
\hline 5 & Compound 3 & 75.34 & 11.71 & 81.35 & No risk & No risk \\
\hline 6 & Compound 4 & 75.34 & 11.71 & 88.52 & No risk & No risk \\
\hline 7 & Compound 5 & 93.84 & 20.68 & 89.03 & No risk & No risk \\
\hline 8 & Compound 6 & 94.34 & 21.36 & 93.53 & No risk & No risk \\
\hline 9 & Compound 7 & 93.84 & 21.22 & 92.94 & No risk & No risk \\
\hline 10 & Compound 8 & 94.96 & 21.33 & 95.16 & No risk & No risk \\
\hline 11 & Compound 9 & 94.96 & 20.28 & 89.84 & No risk & No risk \\
\hline 12 & Compound 10 & 94.96 & 21.33 & 90.17 & No risk & No risk \\
\hline 13 & Compound 11 & 94.35 & 20.72 & 94.33 & No risk & No risk \\
\hline 14 & Compound 12 & 94.36 & 20.39 & 89.08 & No risk & No risk \\
\hline 15 & Compound 13 & 94.36 & 20.39 & 89.08 & No risk & No risk \\
\hline 16 & Compound 14 & 94.36 & 20.39 & 91.38 & No risk & No risk \\
\hline 17 & Compound 15 & 93.98 & 22.00 & 88.36 & No risk & No risk \\
\hline 18 & Compound 16 & 93.98 & 21.00 & 88.08 & No risk & No risk \\
\hline 19 & Compound 17 & 93.98 & 21.53 & 87.55 & No risk & No risk \\
\hline 20 & Compound 18 & 93.98 & 21.73 & 87.49 & No risk & No risk \\
\hline 21 & Compound 19 & 94.49 & 22.67 & 90.43 & No risk & No risk \\
\hline 22 & Compound 20 & 94.49 & 22.49 & 89.08 & No risk & No risk \\
\hline 23 & Compound 21 & 74.67 & 19.76 & 86.53 & No risk & No risk \\
\hline 24 & Compound 22 & 74.67 & 17.91 & 87.74 & No risk & No risk \\
\hline 25 & Compound 23 & 86.12 & 16.00 & 86.44 & No risk & No risk \\
\hline 26 & Compound 24 & 86.12 & 15.32 & 86.45 & No risk & No risk \\
\hline 27 & Compound 25 & 27.79 & 17.27 & 91.32 & No risk & No risk \\
\hline 28 & Compound 26 & 33.28 & 10.83 & 84.48 & No risk & No risk \\
\hline
\end{tabular}


Table 2. Predictive absorption, distribution, and toxicity of curculigoside A and its derivatives (continued)

\begin{tabular}{llccccc}
\hline & & \multicolumn{3}{c}{ Absorption } & Distribution & Toxicity risk parameters \\
\cline { 2 - 6 } No. $\begin{array}{l}\text { Name of } \\
\text { Compound }\end{array}$ & $\begin{array}{c}\text { HIA } \\
(\%)\end{array}$ & $\begin{array}{c}\text { In Vitro Caco-2 } \\
\text { cell permeability } \\
\left(\mathrm{nm} \mathrm{sec}^{-1}\right)\end{array}$ & $\begin{array}{c}\text { In vitro plasma } \\
\text { protein binding }(\%)\end{array}$ & Mutagenicity & carcinogenicity \\
\hline 29 & Compound 27 & 8.02 & 1.88 & 84.37 & No risk & No risk \\
30 & Compound 28 & 79.72 & 10.78 & 82.52 & No risk & No risk \\
31 & Compound 29 & 35.38 & 1.96 & 89.59 & No risk & No risk
\end{tabular}

*Classification: \% human intestinal absorption (\% HIA): 70-100 \% well absorbed, 20-70\% moderately absorbed, 0-20\% poorly absorbed; In Vitro Caco-2 cell permeability ( $\left.\mathrm{nm} \mathrm{sec}^{-1}\right)$ : less than 4 low permeability, between $4-70$ medium permeability, greater than 70 higher permeability; \% plasma protein binding: $>90$ strongly bound, $<90 \%$ weakly bound.

In this study, the Pre-ADMET program was used to predict ADME of curculigoside $\mathrm{A}$ and its derivatives. The aspect prediction of absorption properties included percentage human intestinal absorption (\% HIA) and Caco- 2 cell permeability. Caco- 2 cells are derived from a human colon carcinoma and possess multiple drug transport cycles through the intestinal epithelium. The Caco-2 cells are widely used as an in vitro model for predicting human drug absorption, while HIA is the sum of bioavailability and absorption evaluated from the ratio of excretion or cumulative excretion in urine, bile, and feces. The distribution properties were calculated using Pre-ADMET which will produce predictive plasma protein binding value. Those parameters is important because the degree of plasma protein binding of a drug has an important role on its disposition and the drug's efficacy [7].

\section{Absorption}

All compound in table 1 were predicted to have good absorption properties (\% human intestinal absorption, $\%$ HIA), exception for compounds (25), (26), (27), and (29). Compounds (25), (26), and (29) was predicted to have moderate absorption, while compound (27) have poor absorption proterties. Curculigoside A derivatives has a better absorption than its curculigoside A. Thus, in vitro Caco-2 cell permeability properties of these compounds were predicted to have medium permeability properties; exception for compounds (27) and (29) has low permeability properties.

\section{Distribution}

The compounds of (6), (7), (8), (10), (11), (14), (19), (25), and tamoksifen is strongly bound to plasma proteins while the other compounds are weakly bound to plasma protein. The ability of binding protein plasma affect to drug distribution process.

\section{Toxicity}

The toxicity of curculigoside A and its derivatives was predicted using Toxtree. The curculigoside $\mathrm{A}$ and its derivatives show a negative in mutagenicity and carcinogenicity

\section{Conclusions}

Curculigoside A has absorption properties and permeability at medium level, and is weakly bound to plasma proteins. Aglycone of curculigoside A was predicted to have good absorption properties and its permeability at medium level, and is weakly bound to plasma proteins. Its derivatives, compounds of (2), (3), (4), (5), and (12), showed better absorption and distribution properties than that of curculigoside A (lead). Toxicity Prediction of curculigoside $A$ and its derivatives showed no mutagenic or carcinogenic properties.

\section{Acknowledgement}

This research was supported in part by Riset KK ITB 2014 and Hibah Disertasi Doktor Directorat General of Higher Education 2014.

\section{References}

[1] J. Valls, T. Richard, F. Larronde, V. Leblais, B. Muller, J.C. Delaunay, J.P. Monti, K.G Ramawati, and J.M. Mérillon, Fitoterapia, 77, 2006,416-419.

[2] B.F. Dong, Z.Q. Fang, and J.R. Shi, Zhongguo Zhong Xi Yi Jie He Za Zhi, 26 (Suppl), 2006, 122-125.

[3] S.Y. Lee, M.R. Kim, H.S. Choi, H.I. Moon, J.H. Chung, D.G. Lee, and E.R. Woo, Arch. Pharm. Res., 32, 2009, 1433-1439.

[4] Y.K. Wang, Y.J. Hong, M. Wei, Y. Wu, Z.Q. Huang, R.Z. Chen, and H.Z. Chen, J. Ethnopharmacol., 132, 2010, 233239.

[5] C. Ma, J. Zhang, J. Fu, L. Cheng, G. Zhao, and Y. Gu, Phytother. Res., 25, 2011,922-926.

[6] W. Jiang, F. Fu, J Tian, H. Zhu and J. Hou, Neurosci., 192, 2011, 572-579.

[7] S.K. Lee, I.H. Lee, H.J. Kim, G.S. Chang, and J.E. Chung, Blackwell Publishing Massachusetts, 2003, 418-420.

[8] R. Benigni, C. Bossa, N. Jeliazkova, T. Netzeva, and A. Worth, European Commission Reports, 2008. 